\title{
Nivel de severidad de caries en niños de 5 meses a 3 años que acudieron a la Cátedra de Odontopediatría de la Universidad Católica, Campus Guairá en el periodo 2017-2018
}

\author{
* Ninfa Lucia Jacquett-Toledo , Romina Ramírez-Rolón , Martín Elías Torres-Meza , \\ Karina Alderete-Amarilla \\ Universidad Católica Nuestra Señora de la Asunción "Campus Guaira" \\ Facultad de Ciencias de la Salud. Carrera de Odontología. Paraguay.
}

\begin{abstract}
Cómo referenciar este artículo/
How to reference this article:
\end{abstract}

Jacquett-Toledo NL, Ramírez-Rolón R, TorresMeza ME, Alderete-Amarilla K. Nivel de severidad de caries en niños de 5 meses a 3 años que acudieron a la Cátedra de Odontopediatría de la Universidad Católica Campus Guaira en los periodos 2017 y 2018. Mem. Inst. Investig. Cienc. Salud. 2020; 18(2): 33-38

\begin{abstract}
RE S U ME N
La patología caries sigue siendo una problemática en Paraguay y los niños menores de 5 años se ven afectados con valores altos en cuanto a la frecuencia. Esos valores tienden a aumentar con la edad y las caries inician su aparición en forma cada vez más precoz. Con el objetivo de determinar el nivel de severidad de caries según los criterios de la Organización Mundial de la Salud, evidenciada a través del índice ceo-d, niños de 5 meses a 3 años que acudieron a la Cátedra de Odontopediatría de la Universidad Católica Campus Guairá durante los meses de marzo a setiembre del periodo 2017-2018 fueron evaluado a través de un estudio observacional descriptivo retrospectivo de carácter exploratorio. El muestreo fue no probabilístico de casos consecutivos, y se tuvo como criterio de inclusión a niños con fichas clínicas elaboradas correctamente y en buen estado de conservación. No formaron parte de la muestra los niños cuyos padres no firmaron el consentimiento informado y pacientes ambulatorios. Los resultados mostraron un ceo-d promedio (7/54) de 0,1, iniciándose a los dos años (ceo-d: 0,25 ), aumentando con la edad, siendo 1 el valor del ceo- $d$ a los 3 años, concluyendo que la severidad de caries fue muy baja.
\end{abstract}

Palabras clave: índice ceod, caries dental, odontopediatria, niños.

\section{Level of caries severity in children from 5 months to 3 years who attended the Pediatric Dentistry Service of the Catholic University, Guairá Campus in the period 2017-2018}

\begin{abstract}
The pathology of caries continues to be a problem in Paraguay, children under 5 years are affected by high frequency values and these values tend to increase with age and caries begin to appear earlier and earlier. In order to determine the level of caries severity according to the criteria of the World Health Organization, evidenced by the ceo- $d$ index, children from 5 months to 3 years who attended the Pediatric Dentistry Service of the Catholic University, Guaira Campus from March to September of 2017-2018 were evaluated through a retrospective descriptive observational study of exploratory nature. The sampling was non-probabilistic of consecutive cases, and the criteria for inclusion were children with clinical records correctly prepared and in good condition. Children whose parents did not sign the informed consent and who were outpatients were not included in the sample. The results showed an average ceo-d (7/54) of 0.1 , starting at 2 years (ceo-d: 0.25 ), increasing

Fecha de recepción: diciembre 2019. Fecha de aceptación: junio 2020

* Autor correspondiente: Ninfa Lucía J acquett Toledo. Calle Coronel Romero c/ Las Residentas, N 1241. San $^{\circ}$ Lorenzo - Paraguay. Correo: ninfajacquett@gmail.com. Teléfono: +59521583772 +595983597117
\end{abstract}


with age, with a value of ceo-d of 1 at 3 years, concluding that the severity of caries was very low.

Keywords: ceod index, dental caries, pediatric dentistry, children.

\section{NTRODUCCI ON}

La Organización Mundial de la Salud (OMS) sostiene que la caries afecta a personas de cualquier edad, sexo y raza; teniendo una mayor presencia en sujetos de bajo nivel socioeconómico ${ }^{(1)}$. La caries dental ha ocupado a través de la historia, los primeros lugares entre las 10 primeras causas de consulta de la humanidad y en esta región de las Américas, a pesar de esto y tal vez por no ser una enfermedad que provoque mortalidad, no se le ha dado el lugar que le corresponde de acuerdo a la morbilidad presentada. Otra posible causa es que no se observan avances significativos en los programas de promoción y prevención, en cambio en la parte curativa y de rehabilitación se ven avances científicos ${ }^{(2)}$. Las caries sin tratamiento oportuno afectan la vida de los niños tanto a nivel físico, psicológico y social por lo que el diagnóstico precoz es de relevancia ${ }^{(3)}$.

En Paraguay, un trabajo retrospectivo con datos de investigaciones entre 2000 y 2013 encontradas en universidades de Odontología reveló inicio de caries a los 3 años con un ceo- $d$ de 1,76 , aumentando con la edad y llegando a los 5 años a un ceo- $d$ de $4,73^{(4)}$. En el 2015, una investigación realizada en casa cuna Dr. Carlos Santiviago de la ciudad de Asunción, concluyó que las necesidades de tratamiento odontológicas en niños menores de 6 años se iniciaban a la edad de 1 año e iba en aumento llegando al $100 \%$ en niños de 4 y 5 años. Otro trabajo menciona que la patología caries y por ende la necesidad de obturaciones fue la variable más frecuente en un $93 \%{ }^{(5)}$. En el 2016 un trabajo realizado en una escuela de la ciudad de Capiatá encontró un ceo-d de 3,28 $\pm 3,9$ con un máximo de 16 dientes cariados en niños de 5 años ${ }^{(6)}$. En la ciudad de I tá los resultados de un estudio probabilístico en escuelas públicas realizada en el año 2017 describe que el ceo- $d$ total de la población fue $579 / 133(4,4)^{(7)}$.

La falta de atención oportuna en niños ocasiona una serie de secuelas como son infecciones, problemas estéticos, dificultades para la alimentación, alteraciones del lenguaje aparición de maloclusiones y hábitos orales, además de repercusiones médicas, emocionales y financieras, y las caries son consideradas factor de riesgo para el desarrollo de caries futura en dentición primaria y permanente, así como su asociación con anomalías cardiacas congénitas y endocarditis bacteriana ${ }^{(8,9)}$.

Las autoridades de salud y la profesión odontológica tienen el reto de mejorar la situación de salud bucal de los niños, priorizando aquellos grupos marginados, en quienes se ha concentrado la enfermedad. En este sentido, es necesario identificar la magnitud del problema en grupos poblacionales vulnerables, en donde los factores socioeconómicos y culturales aumentan el riesgo de padecer caries dental ${ }^{(10)}$. Las políticas de salud oral deben estar dirigidas a resolver problemas de salud a nivel comunitario, para el desarrollo y la implementación de programas eficaces ${ }^{(11)}$.

En Paraguay los escolares son el foco de la prevención en programas de salud oral, como el programa "Salvemos al Primer Molar" (2010-2015) dirigido a niños del primer a sexto grado ${ }^{(12)}$.

Como se ve, los datos en la literatura científica revelan altos niveles de caries en niños menores de 5 años, teniendo en cuenta esto, se realizó el presente trabajo, con el objetivo de determinar el nivel de severidad de caries según los criterios de la Organización Mundial de la Salud, evidenciada a través del índice ceo-d en niños de 5 meses a 3 años de edad, que acudieron a la Cátedra de Odontopediatría de la Universidad Católica Campus Guairá durante los meses de marzo a setiembre en el periodo 2017-2018. Los datos podrían ser de alto valor como base para otras investigaciones extensivas a otras poblaciones de la comunidad de la ciudad de Villarrica.

\section{MATERI AL Y METODO}

El diseño del estudio fue observacional descriptivo retrospectivo de carácter exploratorio. Participaron del estudio 54 niños que cumplieron con los criterios de inclusión, es decir, niños en edades comprendidas entre 5 meses a 3 años de edad, de sexo masculino o femenino, con 2 dientes presentes como mínimo, de nacionalidad paraguaya, con fichas clínicas elaboradas correctamente, en buen estado de conservación y que asistieron a la 
clínica de la catedra de Odontopediatría UCV durante los meses de marzo a setiembre del periodo 2017-2018.

Criterios de exclusión: no formaron parte la muestra los niños cuyos padres no dieron su consentimiento, es decir, no firmaron el consentimiento informado y pacientes ambulatorios (en la catedra de Odontopediatria UCV se considera niño ambulatorio al que acude a la clínica una sola vez, por una urgencia o un tratamiento único. Estos procedimientos se registran en una planilla específica llamada: Planilla para paciente ambulatorio).

El muestreo fue no probabilístico de casos consecutivos, pues los sujetos de estudio fueron seleccionados dentro de un intervalo de tiempo específico ${ }^{(9)}$. Para determinar el nivel de caries, según lo observado, se utilizó el índice ceo-d (índice que indica el promedio poblacional de dientes cariados, extraídos/indicados para extracción y obturados en la dentición temporaria(10) y a partir de su promedio se calificó el nivel de severidad de la caries, siguiendo los criterios establecidos por la OMS para el ceo- $d$ y CPOD, considerando un promedio de 0,0 a 1 como muy bajo; 1,2 a 2,6 bajo; de 2,7 a 4,4 intermedio 0 moderado; de 4,5 a 6,5 alto y muy alto $>$ a $6,5^{(13,14,15)}$.

El instrumento de medición estuvo dado exclusivamente por fichas clínicas de la cátedra de Odontopediatría de la UCV.

Para que los niños ingresaran al estudio, se solicitó a los padres que dieran su consentimiento previa explicación detallada de los objetivos del estudio y procedimientos a ser realizados.

En cuanto a los asuntos estadísticos, para el análisis, los datos fueron ingresados en una hoja de cálculo (Microsoft Excel versión 2007). Se organizaron los datos obteniéndose distribuciones de frecuencia de todas las variables de interés en tablas y se utilizó estadística descriptiva.

\section{RESULTADOS}

El 38,2\% de los niños tenía 1 año de edad (Tabla 1 ).

Tabla 1: Distribución de niños según edad. $(n=54)$

\begin{tabular}{|l|l|l|}
\hline Edad & Población & $\%$ \\
\hline menor a 1 año & 14 & 25,9 \\
\hline $\mathbf{1}$ año & 21 & 38,9 \\
\hline $\mathbf{2}$ años & 16 & 29,6 \\
\hline $\mathbf{3}$ años & 3 & 5,6 \\
\hline Total & $\mathbf{5 4}$ & $\mathbf{1 0 0}$ \\
\hline
\end{tabular}

El sexo más frecuente fue el masculino (31/54) (Tabla 2).

Tabla 2: Distribución de niños según sexo. $(n=54)$

\begin{tabular}{l|l|l}
\hline Sexo & Total & \% \\
\hline Masculino & 31 & 57,4 \\
\hline Femenino & 23 & 42,6 \\
\hline Total & $\mathbf{5 4}$ & $\mathbf{1 0 0}$ \\
\hline
\end{tabular}


En la Tabla 3 se observa la presencia de caries en niños a partir de los dos años, siendo el ceo-d de los mismos: 0,25. El ceo-d promedio poblacional (7/54) fue de 0,1. La tabla también revela el predominio del componente cariado.

Tabla 3: Índice ceo- $d$ de niños según edad. $(n=54)$

\begin{tabular}{|c|c|c|c|c|c|}
\hline Edad & Nro de niños & C & E & $\mathbf{0}$ & ceo-d \\
\hline 5 a 11 meses & 14 & 0 & 0 & 0 & 0 \\
\hline 1 año & 21 & 0 & 0 & 0 & 0 \\
\hline 2 años & 16 & 4 & 0 & 0 & 0,25 \\
\hline 3 años & 3 & 3 & 0 & 0 & 1 \\
\hline
\end{tabular}

\section{DISCUSIÓN}

En el presente trabajo, la severidad de caries evidenciada por el índice ceo-d fue muy baja, siendo 0,25 en niños de 2 años, y 1 en niños de 3 años. Datos con valores mayores se observaron en investigaciones como la realizada en la ciudad de San Lorenzo, Paraguay, donde el promedio del índice ceo- $d$ en niños de 2 años fue $1.4 \pm 2,7$ y en niños de 3 años fue $3,4 \pm 3,8^{(10)}$. Este estudio también reporta que en el Hospital Materno Infantil de San Lorenzo en 101 niños 1 a 3 años el ceo-d fue de 2,1. Otra investigación realizada en Escuelas Maternales de San Lorenzo refirió ceo- $d$ de 1,76 en niños de 3 años ${ }^{(16)}$, lo que no coincide con los datos hallados en el presente trabajo de investigación, al reportar niveles de severidad de caries entre bajo a moderado. Estas diferencias podrían deberse al mayor número de muestra en los trabajos mencionados.

Además, el resultado de este estudio $(0,1)$ difiere de resultados hallados en Chile, niños de 2 a 4 años con ceo-d igual a $3^{(13)}$, y a resultados de un estudio realizado en Capiatá, con ceo-d 3,28 $\pm 3,9^{(6)}$ así como también difiere de los resultados encontrados en Itá, ceo-d poblacional de $4,54^{(7)}$. Otro trabajo realizado en Chile con niños de 0 a 3 años reportó datos diferentes con un promedio del ceo-d de $1,31^{(18)}$.

En el presente trabajo la presencia de caries en niños se observó partir de los 2 años. Los datos hallados en la literatura científica refieren que investigaciones realizadas en Facultades de Odontología de Asunción reportaron inicio de caries a los 3 años ${ }^{(4)}$. Otro trabajo realizado en la casa cuna Dr. Carlos Santiviago de Asunción, reportó como edad de inicio 1 año de edad ${ }^{(5)}$. Sería interesante aumentar el tamaño de la muestra, considerando la edad reportada en estos trabajos. Sin embargo, en Lima Perú, se encontraron caries en niños menores de 1 año(8), La diferencia con este trabajo podría estar en que las muestras fueron tomadas en la comunidad, no de niños que acuden la clínica de una Facultad.

Los resultados coinciden con la literatura presentada en que la patología caries aumenta sus valores con la edad y se inicia tempranamente, por lo que es necesario implementar programas enfocados a mejorar la salud oral de niños menores de 2 años. Esta recomendación coincide con la conclusión de dos trabajos que manifiestan la importancia de educar a futuras madres y padres de familia, a fin de tomar los recaudos necesarios mediante medidas preventivas de caries de dental implementadas precozmente ${ }^{(19,20)}$.

Con los resultados observados en este trabajo, se concluyó que la severidad de caries, según criterios de la Organización Mundial de la Salud, evidenciada a través del índice ceo-d en niños de 5 meses a 3 años de edad, que acudieron a la cátedra de Odontopediatría de la UCV durante los meses de marzo a setiembre en el periodo 2017-2018, fue muy baja, se inicia a los 2 años y va incrementándose acorde a la edad.

Teniendo en cuenta este estudio exploratorio, se sugiere la realización de otros trabajos, considerando objetivos (general y específico), diseño, muestreo y tamaño de la muestra con el fin de generalizar los resultados.

\section{REFERENCI AS BI BLI OGRAFI CAS}

1. Agreda $M$, Medina $Y$, Simancas $Y$, Salas $M$, Ablan L. Condiciones de salud periodontales niños en edad escolar.

Act. odontol.Venezolana. [Internet] 2010 [citado J unio 15 2018] 48 (3). Disponible 
en: https: //www.actaodontologica.com/ ediciones/2010/3/art-9/

2. Martínez García M, Marín Hurtado MS, Ceballos Echeverry PT, Sandra Milena Osorio Mendieta SM. Impacto de la calidad en salud oral y caries de la primera infancia. Tesis de Especialización en Administración de la Salud. Universidad Católica de Manizales. [Internet] 2014 [citado Junio 15 2018]. Disponible en: http://repositorio.ucm.edu.co: $8080 /$ jsp ui/ bitstream/handle/10839/751/Monica $\% 20$ Martinez\% 20Garcia.pdf?sequence= 1 \&isAllowed =y

3. Mayor Hernández F, Pérez Quiñones JA, Cid Rodríguez MC, Martínez Brito I, Martínez Abreu J, Moure Ibarra MD. La caries dental y su interrelación con algunos factores sociales. Rev. Med. Electrón. [Internet]. 2014 Jun [citado J unio 15 2018]; 36(3):339-349. Disponible en: http://scielo.sld.cu/scielo.php?script=sc i arttext\&pid=S168418242014000300010\&lng=es.

4. Danei Morel C, Jacquett Toledo NL Frecuencia de caries en niños de 1 a 5 años y conocimientos, actitudes $y$ prácticas de acuerdo a investigaciones realizadas en universidades de Asunción reconocidas por el Ministerio de Educación y Cultura durante los años 2000 al 2013. Revista de la Asociación latinoamericana de Odontopediatría [Internet] 2015 [citado Junio 15 2018]; 5(2):15-25. Disponible en: https: //www. revistaodontopediatria.org /ediciones/2015/2/art-3/

5. Escobar Martínez SV, Jacquett Toledo NL. Necesidades odontológicas en niños de 10 meses a 5 años, que acuden a la casa cuna 1, 2,3 y 4 del Dr. Carlos Santiviago de la ciudad de Asunción. [Trabajo de fin de grado] Asunción: Universidad Autónoma del Paraguay Pierre Fauchard, Facultad de Odontología Pierre Fauchard. 2015.

6. Ruiz Alvarez VM, Jacquett Toledo NL. Nivel de ceo-d según criterios de la OMS y situación del Primer Molar Permanente en niños y niñas de 5 años que acudan al Puesto de Salud "Puerta del Sol" de Capiatá, en febrero y marzo del 2016., Paraguay Oral Research [Internet] 2017 [citado agosto 15 2018]; vol 5 (2) pag: 62 a 78. Disponible

http://paraguayoral.com.py/

7. Alvarez EC, Jacquett Toledo NL Prevalencia y severidad de caries dental en escolares del primer grado de la ciudad de Itá. [Trabajo de fin de grado] Asunción: Universidad Autónoma del Paraguay Pierre Fauchard, Facultad de Odontología Pierre Fauchard. 2017.
8. Villena Sarmiento R, Pachas Barrionuevo $F$, Sánchez Huamán $Y$, Carrasco Loyola M. Prevalencia de caries de infancia temprana en niños menores de 6 años de edad, residentes en poblados urbano marginales de Lima Norte. Rev. Estomatológica Herediana [Internet] 2011 [citado agosto 15 2018]; 21(2): 79-86. https: // www.redalyc.org/pdf/4215/421 539363004.pdf

9. Hulley Stephen B. Cummings Steven. Diseño de Investigaciones Clínicas. 3ra. Edición. . España: Editorial Wolters Kluwer Health.; 2008.

10. Jacquett Toledo NL, Samudio M. Prevalencia de caries en dentición temporal en niños de 1 a 5 años de acuerdo a los criterios ICDAS en el puesto de salud San Miguel de San Lorenzo, Paraguay. Pediatr. (Asunción), [Internet]. 2015 [citado Junio 15 2018]; 42(3): 216-224. http: //dx.doi.org/10.18004/ped. 2015.di ciembre. 216-224.

11. Guedes de Amorim R, Figueiredo MJ, Coelho Leal S, Mulder J, Frencken JE. Caries experience in a child population in a deprived area of Brazil, using ICDAS II. Clin Oral Invest. [Internet] 2012 [citado Junio 15 2018]; 16:51320 doi: 10.1007/s00784-011-0528-9

12. Ministerio de Salud Pública y Bienestar Social (Paraguay) y Organización Panamericana de la Salud. Evaluación del programa "Salvemos al primer Molar y la estrategia PRAT del Ministerio de Salud Pública y Bienestar Social del Paraguay. [Internet] 2012 [citado Junio 15 2018]. Disponible en: https: //www.paho.org/par/index.php?o ption $=$ com_docman\&view $=$ download $\& c$ ategory slüg=publicaciones-concontrapartes\&alias $=387$-evaluacion- delprimer-molar\&I temid $=253$

13. Zaror Sánchez C, Pineda Toledo $P$, Orellana Cáceres J. Prevalencia de Caries Temprana de la Infancia y sus Factores Asociados en Niños Chilenos de 2 y 4 Años. Int. J. Odontostomat $2011 . \quad 5(2): 171-7$. https://scielo. conicyt. cl/scielo.php?scrip $\mathrm{t}=$ sci_arttext\&pid=S0718381Xर̄011000200010

14. Duque C, Mora II. La representación de la epidemiología de la caries en el mundo a través de mapas. Univ Odontol. [Internet] 2012 [citado Junio 15 2018] Ene-Jun. 31(66): 41-50. Disponible en: file:///C:/Users/acer/Downloads/271 0-Texto\% 20del\% 20art\% C3\% ADculo11233-1-10-20120807.pdf

15. Vázquez Rodríguez SB, Bayardo González RA, Alcalá Sánchez JA, Maldonado MA. Prevalencia y severidad 
de caries dental en niños de 0 a 12 años. Rev Tamé [Internet] 2016 [citado 20 Julio 2020]; 5 (13): 459$462 . \quad$ Disponible en: http://www.uan.edu.mx/d/a/publicacio nes/revista_tame/numero_13/Tam1613 -05i.pdf

16. Noguera Riveros A, Jacquett Toledo N L. Prevalencia de la succión no nutritiva e índice de caries en niños de escuelas maternales de la ciudad de San Lorenzo. 2011. [Trabajo de fin de grado] Asunción: Universidad Autónoma del Paraguay Pierre Fauchard, Facultad de Odontología Pierre Fauchard; 2012

17. Páez Caballero M H. Perdida prematura de dientes temporarios anteriores en niños que concurrieron a la cátedra de Odontopediatria de la clínica integrada Pierre Fauchard de marzo a noviembre del año 2010. [Trabajo de fin de grado] Asunción: Universidad Autónoma del Paraguay Pierre Fauchard, Facultad de Odontología Pierre Fauchard; 2013

18. Núñez F L, Sanz B J, Mejía L G. Caries dental y desarrollo infantil temprano: Estudio piloto. Rev. chil. pediatr. [Internet]. $2015 \quad$ Feb [citado 20 Julio 2020 ] ; 86(1): 38-42. Disponible en: https: //scielo.conicyt.cl/scielo.php?scrip $\mathrm{t}=\mathrm{sci}$ arttext\&pid=S0370-

$4106 \overline{2} 015000100007 \&$ lng =es. http://d x.doi.org/10.1016/j.rchipe.2015.04.007
19. Zaror Sánchez C, Pineda Toledo P, Orellana Cáceres J. Prevalencia de caries temprana de la infancia y sus factores asociados en niños chilenos de 2 y 4 años. Int. J. Odontostomat. [Internet] 2011[citado 20 Julio 2018]; 5(2):171-177. Disponible en:

https: //scielo. conicyt.cl/scielo.php?scrip $\mathrm{t}=\mathrm{sci}$ arttext\&pid=S0718-

$381 \times \overline{2} 011000200010 \&$ Ing $=$ es. http: //d x.doi.org/10.4067/S0718381X2011000200010.

20. Alvarez Caballero LR, Jacquett Toledo NL, Chirife MT. Índice de caries en escolares y grado de conocimiento y práctica de los padres en relación a la prevención bucodental de sus hijos en dos escuelas de la ciudad de Santa Rita durante el año lectivo. [Trabajo de fin de grado] Asunción: Universidad Autónoma del Paraguay Pierre Fauchard, Facultad de Odontología Pierre Fauchard. 2011 